\title{
The relationship between time to surgery after neoadjuvant chemotherapy and survival in breast cancer patients:a meta-analysis
}

\section{YongCheng Su ( $\sim$ syczb2643937537@163.com )}

Xiamen University and Fujian Medical University Affiliated First Hospital https://orcid.org/0000-00016322-7681

\section{XiaoGang Zheng}

Xiamen University and Fujian Medical University Affiliated First Hospital

\section{Research}

Keywords: Breast Cancer, Time to Surgery, Survival, Meta-Analysis

Posted Date: October 30th, 2020

DOl: https://doi.org/10.21203/rs.3.rs-44882/v2

License: (c) (i) This work is licensed under a Creative Commons Attribution 4.0 International License.

Read Full License 


\section{Abstract}

PURPOSE: This meta-analysis aims to evaluate the impact of delaying surgery in operable breast tumor patients after neoadjuvant chemotherapy (NAC) on survival.

METHODS:An electronic literature retrieval was conducted on PubMed/Medline and EMBASE((between January 2000 and June 2020). The primary end point was overall survival(OS),secondary end points included disease-free survival (DFS) or recurence-free survival (RFS). The HR with $95 \%$ confidence intervals were calculated using a random-effects or fixed-effects model.

RESULTS:The combined HR for OS was 1.51 (95\% Cl:1.30-1.76; $\mathrm{P}=0.000)$ by fixed effects model.No statistically significant heterogeneity was found $(P=0.168, I 2=31.3 \%)$. The pooled HR for RFS/DFS was $1.59(95 \% \mathrm{Cl}: 1.30-1.95, \mathrm{I} 2=66.0 \%)$ by random-effects model,with significant heterogeneity.

CONCLUSION:Our meta-analysis revealed a significant adverse association between longer TTS after NAC and more inferior OS and RFS/DFS in patients with breast cancer.Clinicians and patients should minimize surgical delay after NAC as much as possible.

\section{Background}

Breast tumor is the most common malignancy in women today,and surgical treatment remains the primary treatment in most patients with breast cancer.Approximately $37-40 \%$ of breast cancer patients receive surgery[1].Most patients begin adjuvant chemotherapy (AC) within a few weeks after breast surgery. However it is unknown whether delayed AC would result in poorer outcomes for breast cancer patients.Several studies have explored the appropriate timing of adjuvant chemotherapy for operative patients with breast cancer,and there is still no consensus on the optimal timing of postoperative AC for breast cancer patients.A systematic review and meta-analysis demonstrated that a longer waiting-time from surgery to AC would lead to worth outcomes,especially in triple-negative breast cancer (TNBC) patients[2].

A variety of clinical trials demonstrated that neoadjuvant chemotherapy (NAC) is a well-established treatment option for patients with locally advanced or inoperable breast tumors[3].Furthermore,both earlystage and advanced breast tumors can benefit from NAC to increasing breast conservation rates[4]. While there have been few studies investigated the optimal operative time after NAC for patients with operable breast cancer.Our study aims to explore the impact of delaying surgery in operable breast tumor patients after NAC on survival.

\section{Materials And Methods}

Data source:An electronic literature retrieval was conducted on PubMed/Medline and EMBASE((between January 2000 and June 2020).Search terms were used such as "timing to surgery" or "time to surgery" and "breast neoplasm" and "survival" published in English. 
Selection criteria:The included literature needs to meet the following criteria:1)All of the patients received preoperative NAC,the time interval from NAC to surgery was documented.2)The relationship between the time from NAC to surgery and survival should be reported.3)The primary end point was overall survival(OS),secondary end points included disease-free survival (DFS) or recurrence-free survival (RFS),4)The hazard ratio (HR) with $95 \%$ confidence intervals (Cls) should be reported directly or sufficient data was provided to calculate them.To avoid publication bias,both the full-text and the summary of the meeting were included in our analysis.Patients would be excluded if they had received AC therapy,had stage IV disease, or had undergone mastectomy at an outside hospital.

\section{Data extraction and quality assessment:}

Two experienced authors((S.Y.C and Z.X.G) independently extracted data from the included studies. The data extracted from the literature included: first author's name,publication year and country,population characteristics, waiting time,HR with corresponding $95 \% \mathrm{Cl}$,and covariates in the fully adjusted model.RFS were treated as DFS in our analysis because the definition of RFS was similar to DFS[2]. Waiting time was defined the time interval from the end of preoperative NAC to initiation of surgery.Several studies had revealed that the earlier surgery was performed after preoperative NAC,the better outcome the patients would get.What's more,due to the impact on survival that caused by a too-short delay (i.e.one week) would so small that could not be observed.Due to a lack of original data for articles included in our study,we could not calculate the best cut-off of time intervals between surgery and NAC.We used 4-weeks as cut-off in our meta-analysis,for there were six of nine studies used 4 weeks as reference in our metaanalysis.

We used the Newcastle-Ottawa Scale (NOS) criteria to assess the quality of all included studies[5].Four studies showed high quality ( $\geq 8$ points)[6],and all studies scored six points or more according to the NOS criteria,indicating the quality of all included studies was good[7].Any disagreements on the quality assessment and data extraction would be resolved by consensus or consultation of a third party.

\section{Statistical analysis:}

We used the software Stata 15.0 to perform statistical analyses.And the $\mathrm{I}^{2}$ statistic and the Q-test were used to assess statistical heterogeneity. When $P>0.1$ and $\mathrm{I}^{2}<50 \%$,we used the fixed-effects model, once $\mathrm{P}<0.1$ and $\mathrm{I}^{2}>50 \%$,the random-effects model was used[8]. Our analysis used funnel plots, the Begg and Egger tests to assess publication bias.Publication bias would be considered exist when the funnel plot was asymmetrical.What's more,we also used Begg and Egger tests to evaluated whether the funnel plot was asymmetrical,when P-value of Begg or Egger was $<0.05$, suggested that there was publication bias in all studies $[9,10]$.For all the statistical analyses, $p<0.05$ was considered statistically significant.

\section{Results}


Data synthesis:A total of four hundred and five potentially relevant publications were found according to our initial search strategy,one hundred and sixteen publications from PubMed/Medline,two hundred and eighty-nine publications from Embase. There were three hundred and one publications after duplicate publications were removed; of these,we excluded two hundred and sixty-eight articles because the title or abstract did not meet the eligibility criteria.Eight systematic reviews,four studies on metastatic breast cancer,and eleven non-neoadjuvant chemotherapy were further excluded. Eventually,nine studies were identified as eligible for our analysis,including nine independent studies for OS[11-19],six studies for RFS[11,12,15-18],and three for DFS[13,14,19]. The flow chart of the literature search and study selection is shown in figure 1.

Study quality:The eligible studies were conducted in Australia,Saudi Arabia, Italy,Peru,the USA and Canada. The number of participants ranged from 58-1101 per study for 4755 patients across the nine studies.Detailed baseline characters of each eligible study were listed in table 1.And table 2 showed the HR results from each eligible study.

Data synthesis:The combined HR for OS was 1.51 (95\% Cl:1.30-1.76,P=0.000) by fixed-effects model.No statistically significant heterogeneity was found $\left(P=0.168 ; I^{2}=31.3 \%\right)$, and this difference was statistically significant $(Z=4.70 ; p=0.000)$, figure 2 .

The pooled HR for RFS/DFS was 1.59 (95\% Cl:1.30-1.95, ${ }^{2}=66.0 \%$,figure 3 a) by random-effects model,with significant heterogeneity. When the study by T.L. Sutton et al. [12] which contributed substantial heterogeneity was excluded,low heterogeneity was found $\left(P=0.197, I^{2}=29.0 \%\right)$, the pooled HR was 1.39 (95\%Cl:1.21-1.58) by fixed-effects model,the data was statistically significant $(Z=4.16$, $p=0.000)$, figure $3 b$.

Subsequently,subgroup analysis was performed based on different time intervals. In our subgroup analysis, patients who received surgery with TTS $>4$ weeks had a worse prognosis.Using TTS < 4 weeks as a reference time interval,the pooled HRs for OS and DFS/RFS were 1.39 (95\%Cl:1.18-1.63) and1.33(95\%Cl:1.16-1.53),respectively. In terms of DFS/RFS, as shown in figure 5 , there was no different in the patients who underwent surgery in 4-6weeks or > 6 weeks, the combined HRs were 1.24 (95Cl\%:0.69$\left.2.22,1^{2}=92.8 \%\right)$ and $1.84\left(95 \mathrm{Cl} \%: 0.89-3.79, \mathrm{I}^{2}=81.2 \%\right)$,respectively, and the differences did not reach statistical significance.As for OS,the pooled HRs for the breast cancer patients who underwent surgery with TTS 4-6weeks and $>6$ weeks were $0.87(95 \% \mathrm{Cl}$ : $0.67-1.10)$ and 1.34 ((95\% Cl:1.04-

1.71),respectively.As can be seen,two studies explored the relationship between TTS 4-6 weeks and OS $[13,18]$, the pool HR revealed that patients with TTS $4-6$ weeks reduced in the risk of death by $13 \%$,with no significant heterogeneity $\left(I^{2}=0 \%, P=0.69\right)$, while the difference was not statistically significant,so the results should be interpreted cautiously. The pooled HR for the breast cancer patients who underwent surgery with TTS > 8 weeks were $1.49\left(95 \% \mathrm{Cl}: 1.05-2.12,1^{2}=0 \%\right)$, and this difference was statistically significant(figure 4). 
Sensitivity analysis was used to assess the root of heterogeneity.As shown in figure 6,the individual data set had no significant influence on the OS and DFS/RFS, demonstrating the reliability and stability of the results in our meta-analysis.

Asymmetrical funnel plot showed in figure $7 \mathrm{a}$ and $\mathrm{P}=0.001<0.05$ for Egger's test demonstrated that our studies existed publication bias for OS (figure 8a). While statistical tests showed no publication bias for RFS/DFS was found in our study( $P=0.93>0.05$ for Egger's test and $p=0.048$ for Begg's test),figure 8 b.Then,trim-and-fill method was conducted to adjust funnel plots[20,21].As evidenced in figure 9 ,four missing studies were added in analysis of OS.The recalculated results were still significant for OS, the HR was 1.39 (95\% Cl:1.11-1.75), indicating the conclusions in our meta-analysis were stable and reliable.

\section{Discussion}

The meta-analysis showed that TTS delays after NAC was positively connected with the risk of death in patients with operable breast cancer.The combined HR demonstrated that OS and RFS/DFS were significantly worse in patients with TTS > 4-weeks compared to patients with TTS < 4-weeks $(H R=1.51, P=0.000$ and $H R=1.39, P=0.000$, respectively). Therefore, it is responsible for considering that a 4weeks delay in surgery after NAC is connected with an increase in the relative risk of death of approximately $51 \%$ for OS, and $39 \%$ for RFS/DFS.

As shown in figure 7,the asymmetrical funnel plot revealed a publication bias for OS in our metaanalysis.After adjusted by trim-and-fill method,the corrected results of OS was still statistically significant $(H R=1.39 ; p<0.01)$,indicating our results were robust and reliable.

It is well known that breast cancer has many distinct molecular subtypes,and different subtypes have totally different clinical behaviors[22], of course,their prognoses are also significant difference[23]. Whether the timing of surgery after NAC has an impact on the prognosis of different subtypes of breast cancer. The study by Rachel A. Sanford revealed that there was no distinct difference between different subtypes[18], while due to the number of breast cancer patients with different subtypes in their study varied greatly, their finding were not representative and should be considered cautiously. The study by LP Rebaza et al. showed that there were not considerable differences in the survival curves by molecular subtypes,except for luminal B subtypes[15].The study done by Kausar Suleman et.al demonstrated that patients would achieve a longer survival time and a better PCR rate if they underwent surgery within 8 weeks after NAC,especially in patients with ER+/HER-2+ subtype.Besides well-known molecular subtypes,obesity is also an independent prognostic factor for breast cancer patients[24], which would cause a shorter OS and a worse distant recurrence-free survival[25].

Previous guidelines indicated that 2-4 weeks after NAC might be the best timing of surgery[25],the studies by Cletus Arciero[13]and RachelA.Sanford[18] demonstrated that a longer TTS was connected with a worse prognosis,compared with TTS $<8$ weeks, the combined HR for OS was 1.49 (95\%Cl:1.05$2.12, P=0.025$, figure 4 ), whether these conclusions were related to a longer waiting time would lead to 
tumor growth.Ladd J. Turner and his colleagues compared the size of the tumor on USS-6 and the pathology report to explore whether delayed surgery after NAC would lead to an increase in tumour size,and then they found that there were not apparent growth in tumor size between TTS $<6$-weeks and TTS > 6-weeks[26],Wagner, Jamie L et al. also found the same conclusion in their study[27].

Our meta-analysis had some limitations. Firstly,our finding was based totally on observational studies,we could not completely rule out the effect of confounding on our analysis. Therefore,the conclusion should be interpreted cautiously.It is unethical and unrealistic to carry out a randomized controlled clinical trial in patients to explore the effect of delaying surgery after neoadjuvant chemotherapy on overall survival,therefore,we believe that it may be the only valid method for exploring the answer such a question.Secondly,due to individual information not available, subgroup analyses according to different features failed.Thirdly, different phenotypes of breast cancer would lead to uncertain prognosis;in part,survival is dependent upon breast cancer subtypes [28]. However,because of insufficient information provided in the original studies, we did not further explore whether TTS delays after NAC had an impact on the prognosis of breast cancer patients with different hormone receptor and HER2 status.Despite these limitations,our meta-analysis still has crucial clinical directive significance.Physicians should minimize surgical delay after NAC for breast cancer patients when possible.

\section{Conclusion}

Our meta-analysis revealed a significant adverse association between longer TTS after NAC and more inferior OS and RFS/DFS in patients with breast cancer.Clinicians and patients should minimize surgical delay after NAC as much as possible.

\section{Declarations}

Ethical approval and consent to participate : All procedures performed in studies involving human participants were in accordance with the ethical standards of the institutional research committee and with the 1964 Helsinki declaration and its later amendments or comparable ethical standards.

Consent for publication:Not applicable

Availability of data and materials:Not applicable

Informed consent :The study was approved by the local institutional review board, and the requirement for informed consent was waived because of the retrospective nature of this study.

Competing interests:The authors declare that they have no competing interests.

Funding :This research did not receive any specific grant from funding agencies in the public, commercial, or not-for-profit sectors. 
Authors' contributions: S.Y.C and Z.X.G collated data and did analyses. S.Y.C planned and performed analyses,and was a major contributor in writing the manuscript. All authors read and approved the final manuscript.

\section{Acknowledgements: Not applicable}

\section{References}

1. Cui L, Fan P, Qiu C, Hong Y: Single institution analysis of incidence and risk factors for postmastectomy pain syndrome. Scientific reports 2018;8:11494.

2. Zhan QH, Fu JQ, Fu FM, et al.: Survival and time to initiation of adjuvant chemotherapy among breast cancer patients: a systematic review and meta-analysis. Oncotarget 2018;9:2739-2751.

3. Kurozumi S, Inoue K, Matsumoto $\mathrm{H}$, et al.: Prognostic utility of tumor-infiltrating lymphocytes in residual tumor after neoadjuvant chemotherapy with trastuzumab for HER2-positive breast cancer. Scientific reports 2019;9:1583.

4. Wu J, Cao G, Sun X, et al.: Intratumoral Spatial Heterogeneity at Perfusion MR Imaging Predicts Recurrence-free Survival in Locally Advanced Breast Cancer Treated with Neoadjuvant Chemotherapy. Radiology 2018;288:26-35.

5. Stang A: Critical evaluation of the Newcastle-Ottawa scale for the assessment of the quality of nonrandomized studies in meta-analyses. Eur J Epidemiol 2010;25:603-605.

6. Braithwaite D, Walter LC, Izano M, Kerlikowske K: Benefits and Harms of Screening Mammography by Comorbidity and Age: A Qualitative Synthesis of Observational Studies and Decision Analyses. Journal of general internal medicine 2016;31:561-572.

7. Deng $X$, Wang $L$, Yang T, et al.: A meta-analysis of diffusion tensor imaging of substantia nigra in patients with Parkinson's disease. Scientific reports 2018;8:2941.

8. Higgins JPT, Thompson SG, Deeks JJ, Altman DG: Measuring inconsistency in meta-analyses. BMJ 2003;327:557.

9. Egger M, Smith GD, Schneider M, Minder C: Bias in meta-analysis detected by a simple, graphical test. BMJ 1997;315:629.

10. Begg CB, Mazumdar M: Operating Characteristics of a Rank Correlation Test for Publication Bias. Biometrics 1994;50:1088-1101.

11. Suleman K, Almalik O, Haque E, et al.: Does the Timing of Surgery after Neoadjuvant Therapy in Breast Cancer Patients Affect the Outcome? Oncology 2020;98:168-173.

12. Sutton TL, Schlitt A, Gardiner SK, et al.: Time to surgery following neoadjuvant chemotherapy for breast cancer impacts residual cancer burden, recurrence, and survival. Annals of Surgical Oncology 2020;27:S9-S10.

13. Arciero C, Buhariwalla K, Liu Y, et al.: Time from Completion of Neo-adjuvant Chemotherapy to Surgery: Effects on Outcomes in Breast Cancer Patients. The Breast Journal 2020. 
14. Lai V, Hajjaj O, Chia S, Simmons C: "Needle-to-knife wait time and impact on pCR in patients undergoing neoadjuvant chemotherapy for breast cancer.", 2019.

15. Rebaza LP, Galarreta JA, Castañeda C, et al.: Impact of the time interval between neoadjuvant chemotherapy and surgery in Latin-Americans breast cancer patients. Cancer Research 2019;79.

16. Cinausero M, Galli G, Basile D, et al.: Prognostic value of the interval between the last neoadjuvant chemotherapy (CT) administration and breast surgery: Preliminary data of the NeoBracer international observational study. Journal of Clinical Oncology 2018;36:e12629.

17. Omarini C, Guaitoli G, Noventa S, et al.: Impact of time to surgery after neoadjuvant chemotherapy in operable breast cancer patients. Eur J Surg Oncol 2017;43:613-618.

18. Sanford RA, Lei X, Barcenas CH, et al.: Impact of Time from Completion of Neoadjuvant Chemotherapy to Surgery on Survival Outcomes in Breast Cancer Patients. Ann Surg Oncol 2016;23:1515-1521.

19. Piacentini F, Filieri ME, Grizzi G, et al.: Impact of time to surgery after neoadjuvant chemotherapy in patients with operable breast cancer. Annals of Oncology 2015;26:i4-i5.

20. Yen $C$, Hung $M$, Wong $Y$, et al.: Role of intravenous immunoglobulin therapy in the survival rate of pediatric patients with acute myocarditis: A systematic review and meta-analysis. Scientific reports 2019;9:10459.

21. Zhou L, Cai X, Liu Q, et al.: Prognostic Role of C-Reactive Protein In Urological Cancers: A MetaAnalysis. Scientific reports 2015;5:12733.

22. Tao M, Song T, Du W, et al.: Classifying Breast Cancer Subtypes Using Multiple Kernel Learning Based on Omics Data. Genes 2019;10:200.

23. Schatlo B, Fandino J, Smoll NR, et al.: Outcomes after combined use of intraoperative MRI and 5aminolevulinic acid in high-grade glioma surgery. Neuro-oncology 2015;17:1560-1567.

24. Chan D, Vieira AR, Aune $D$, et al.: Body mass index and survival in women with breast cancersystematic literature review and meta-analysis of 82 follow-up studies. Ann Oncol 2014;25:19011914.

25. Thill M, Jackisch C, Janni W, et al.: AGO Recommendations for the Diagnosis and Treatment of Patients with Locally Advanced and Metastatic Breast Cancer: Update 2019. Breast Care 2019;14:19.

26. Ladd S, Turner J: Neoadjuvant Chemotherapy in Breast Cancer: Does Delayed Surgery Result in Tumour Progression? Clinical Oncology 2017;29:e100.

27. Wagner JL, Warneke CL, Mittendorf EA, et al.: Delays in primary surgical treatment are not associated with significant tumor size progression in breast cancer patients. Annals of Surgery 2011;254:119124.

28. Chia SK, Bramwell VH, Tu D, et al.: A 50-gene intrinsic subtype classifier for prognosis and prediction of benefit from adjuvant tamoxifen. Clin Cancer Res 2012;18:4465-4472. 


\section{Tables}

Please see the supplementary files section to view the tables.

Figures

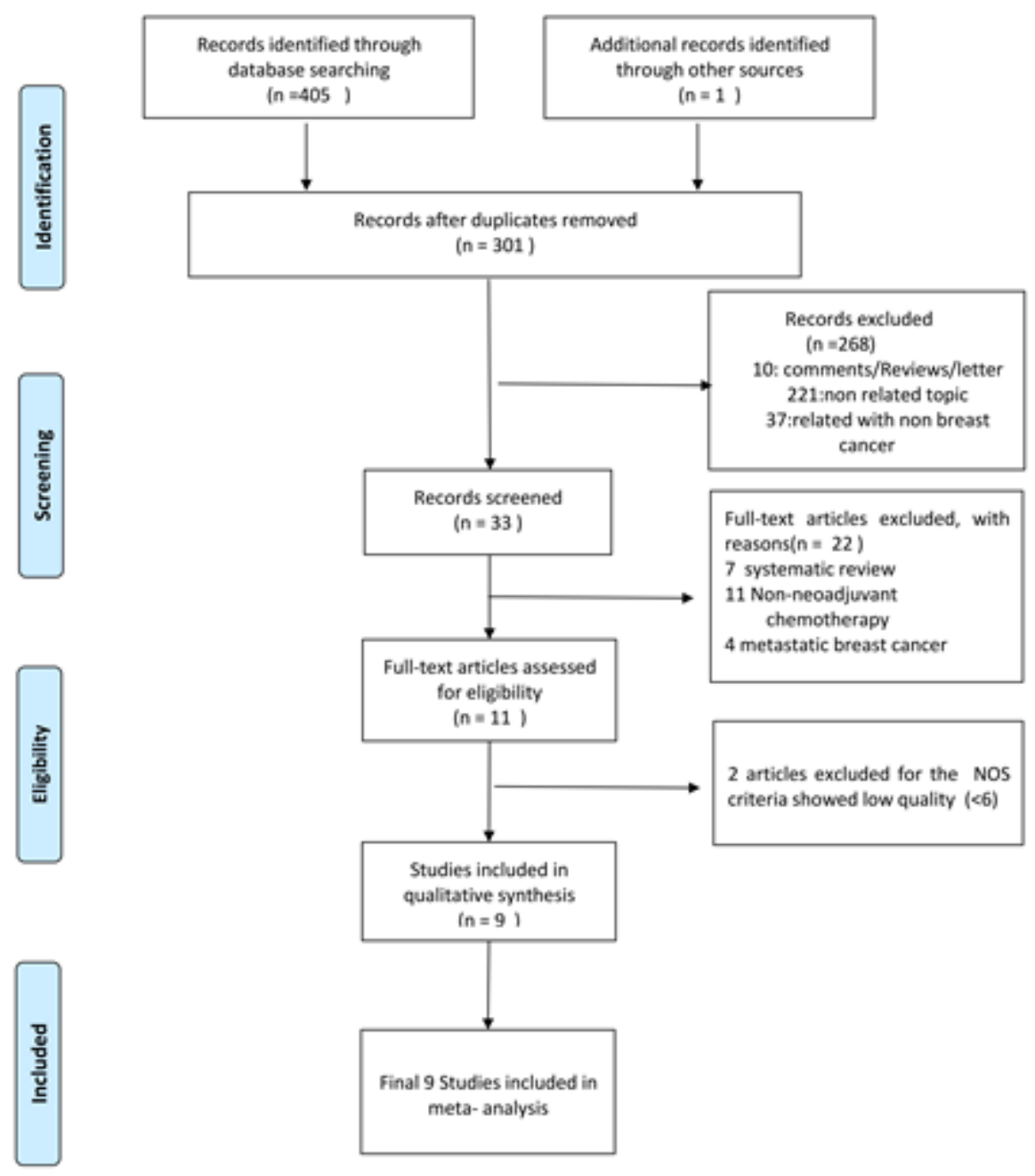

Figure 1

Flowchart of the study selection strategy 
ES $(95 \%$ Cl)

$(D+L)$

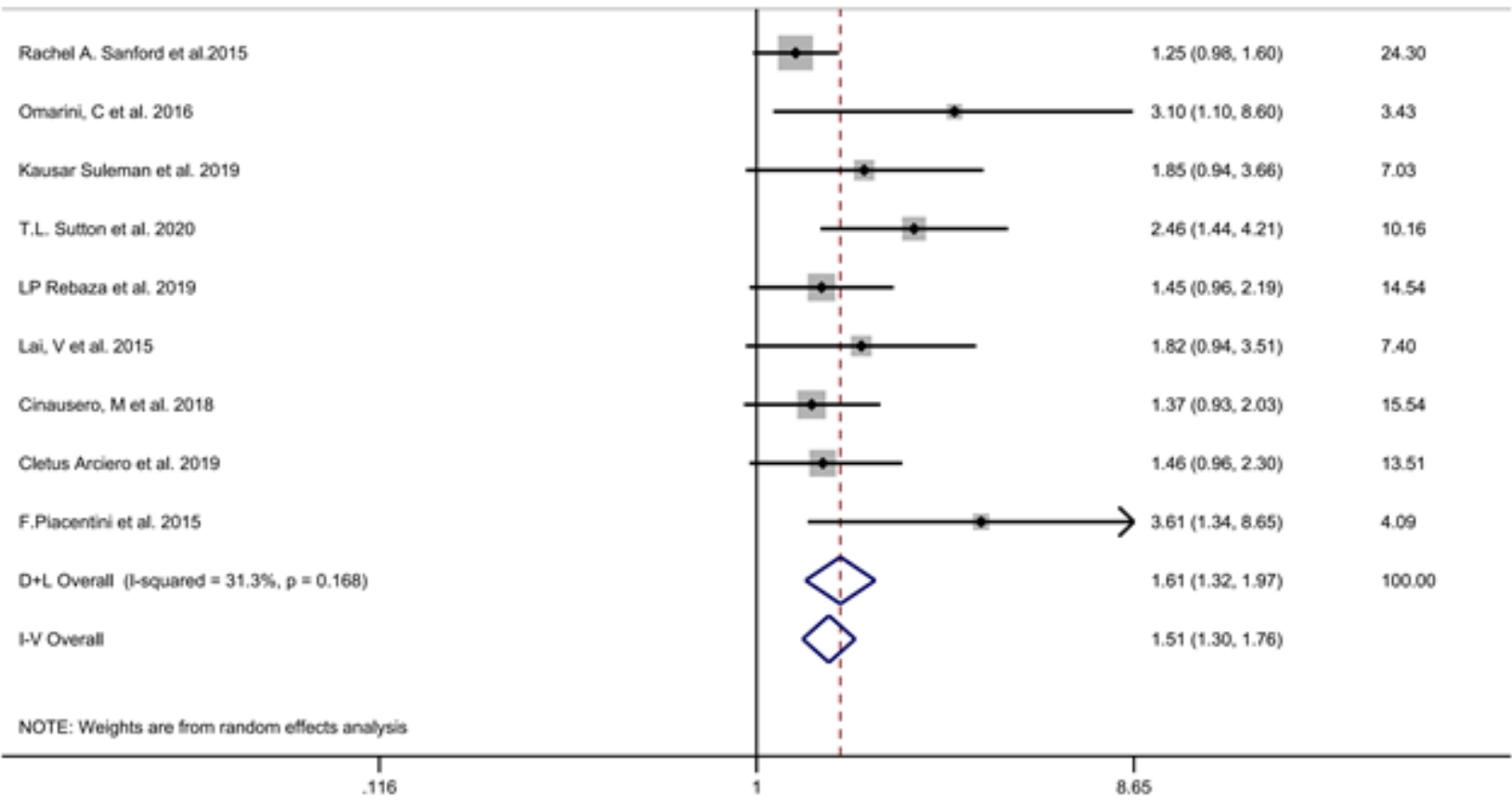

\section{Figure 2}

The association between 4-weeks surgical delay and OS. 
a

\begin{tabular}{|c|c|c|c|}
\hline & & & \\
\hline 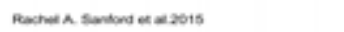 & & 120 cosen, $1.50 \%$ & seos \\
\hline Onam cetas ans & & $2 n(1,3 a>n)$ & 4.63 \\
\hline 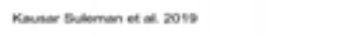 & & $1.48(0.000 .228)$ & $\sin$ \\
\hline rL sumon et at 2000 & $\rightarrow$ & $200(1,22,229)$ & san \\
\hline SP Retara et ac sost & & 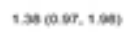 & 1200 \\
\hline La. $v$ enden zons & & $2.15(0.00 .495)$ & $4 \pi$ \\
\hline Clons Aviero et a. 2019 & & $900097,9,73)$ & 5462 \\
\hline Chanera Met ac 2010 & & 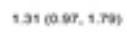 & n4:12 \\
\hline 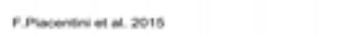 & & $\sin (1.42 .095)$ & 362 \\
\hline 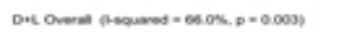 & & $1.90(1,3 a, 198)$ & $=0000$ \\
\hline w woverat & & 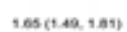 & \\
\hline 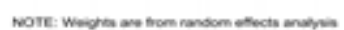 & & & \\
\hline
\end{tabular}

b

\begin{tabular}{|c|c|c|c|}
\hline 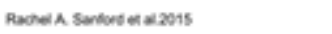 & $\rightarrow$ & 1285 (ase 1.99$)$ & $n \infty$ \\
\hline $\operatorname{Cram}(\cot \alpha 20016$ & & $2.11(1.95,7.17)$ & 203 \\
\hline 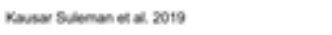 & & $2.480 .96 .228)$ & 1194 \\
\hline 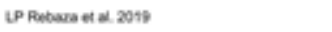 & & 1.36 (1997. 1968) & s.31 \\
\hline La. Veat 2015 & & $2.1510 .95 .4 .95)$ & 291 \\
\hline Ches Avien ex a. 2019 & & $130(097.193)$ & 598 \\
\hline Chenera Net 2.2018 & & 131097.490 & usa \\
\hline 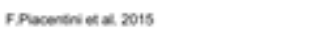 & & $3 \pi(1.22 .990)$ & 200 \\
\hline 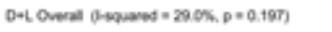 & & $\cos (12 x, 1,00)$ & 900,00 \\
\hline WOwat & & $139(120,1960)$ & \\
\hline 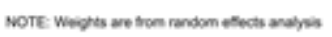 & & & \\
\hline
\end{tabular}

\section{Figure 3}

a: The association between 4-weeks surgical delay and RFS/DFS b: The association between 4-weeks surgical delay and RFS/DFS after the study by T.L.Sutton was excluded. 

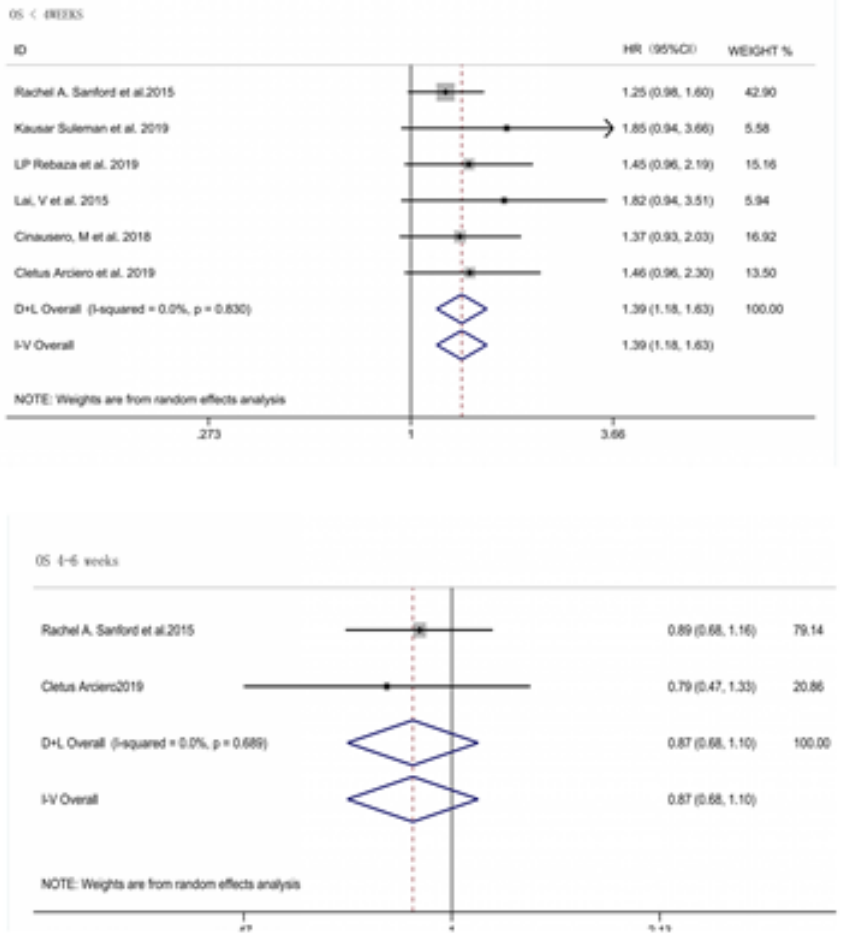

\section{Figure 4}

Subgroup analysis by different time intervals for OS.

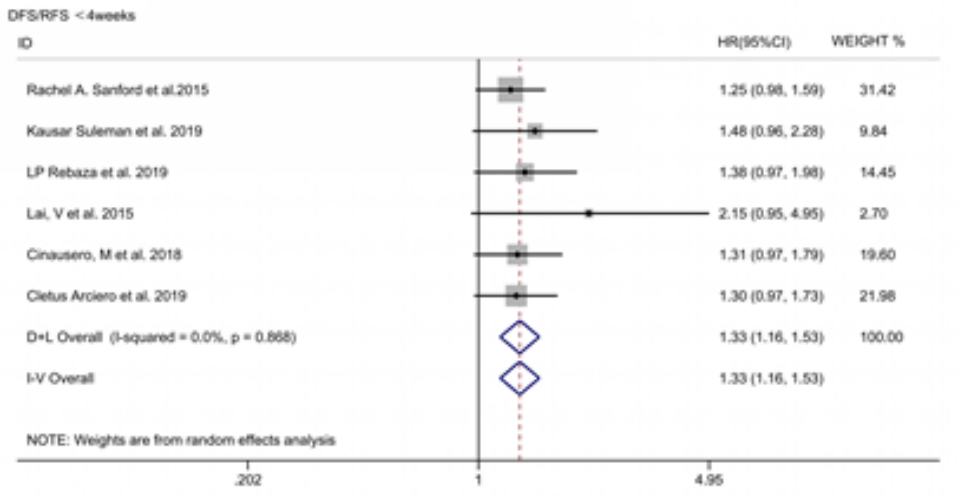

DFSPFS 4-WEeKs

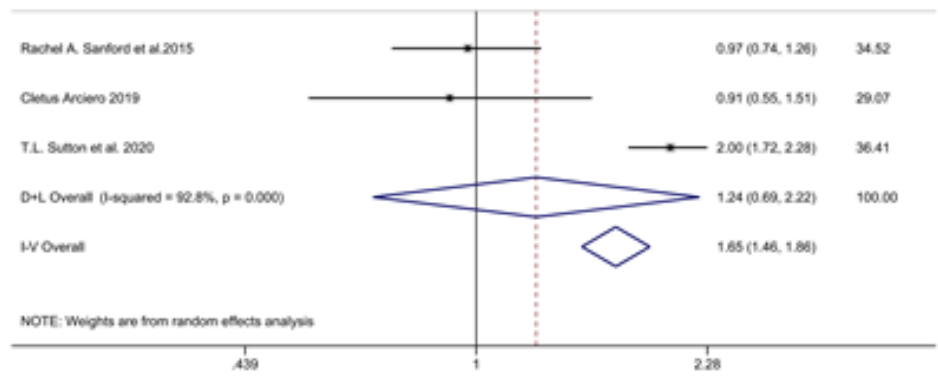

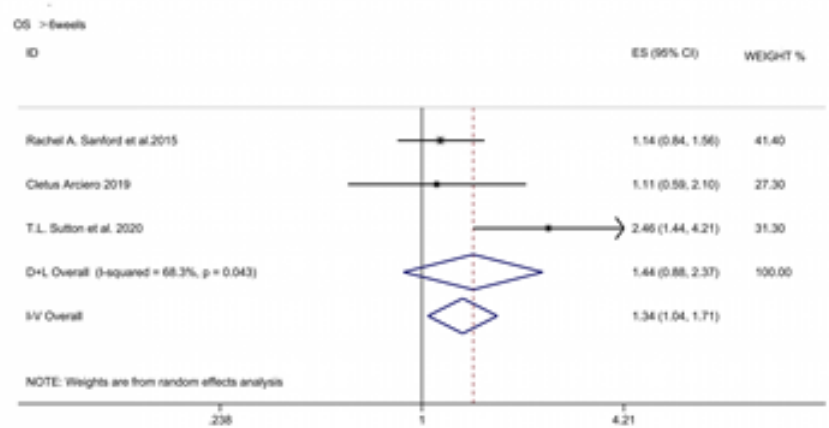

as > soents

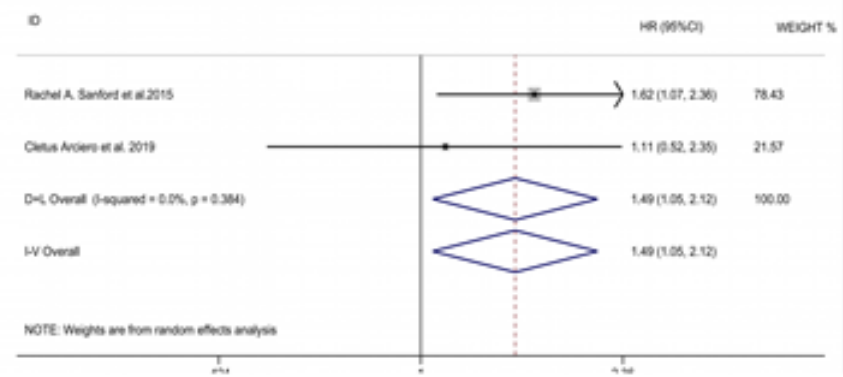

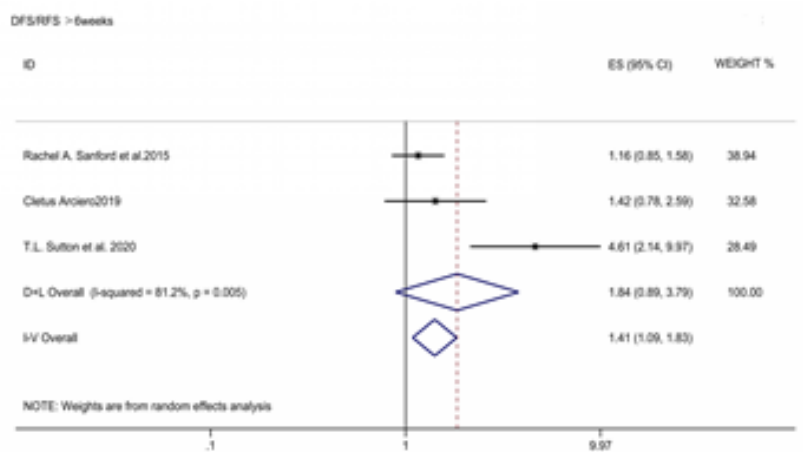

Figure 5 
Subgroup analysis by different time intervals for DFS/RFS.

a

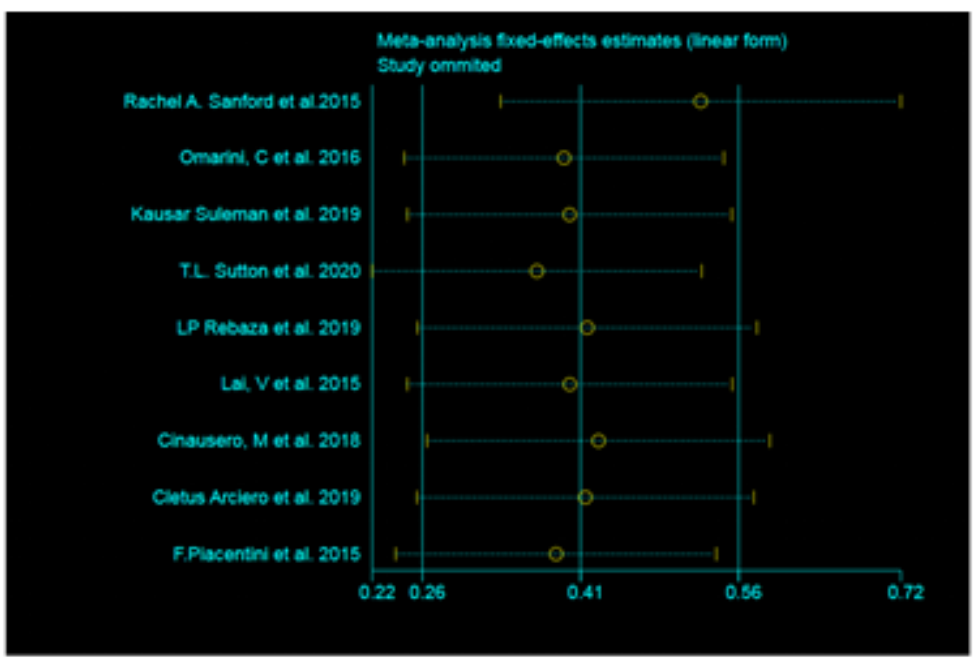

b

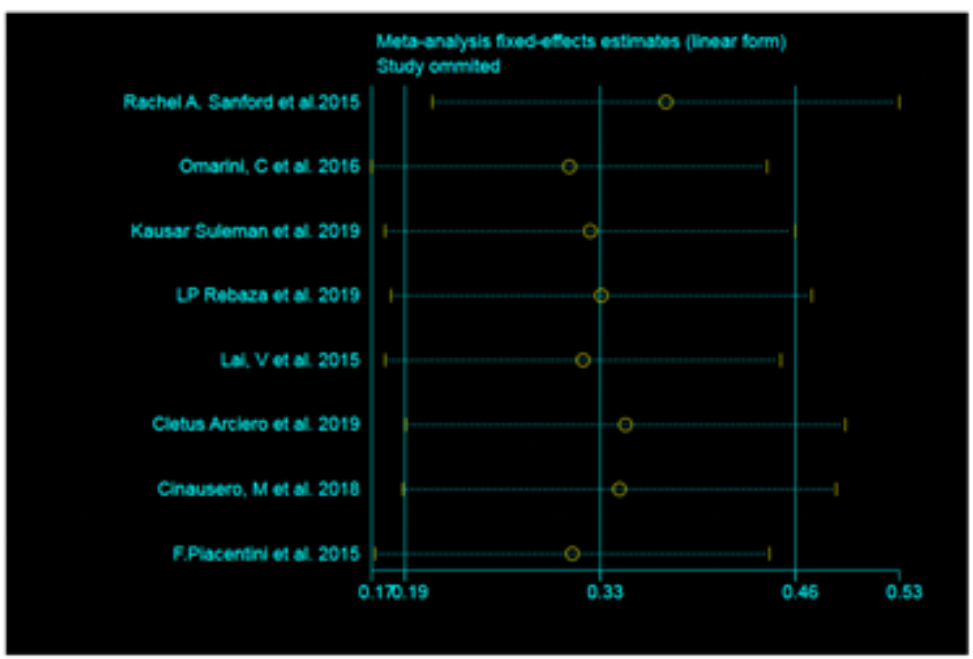

Figure 6

Sensitivity analyses for included studies concerning OS(a) and RFS/DFS(b). 
a
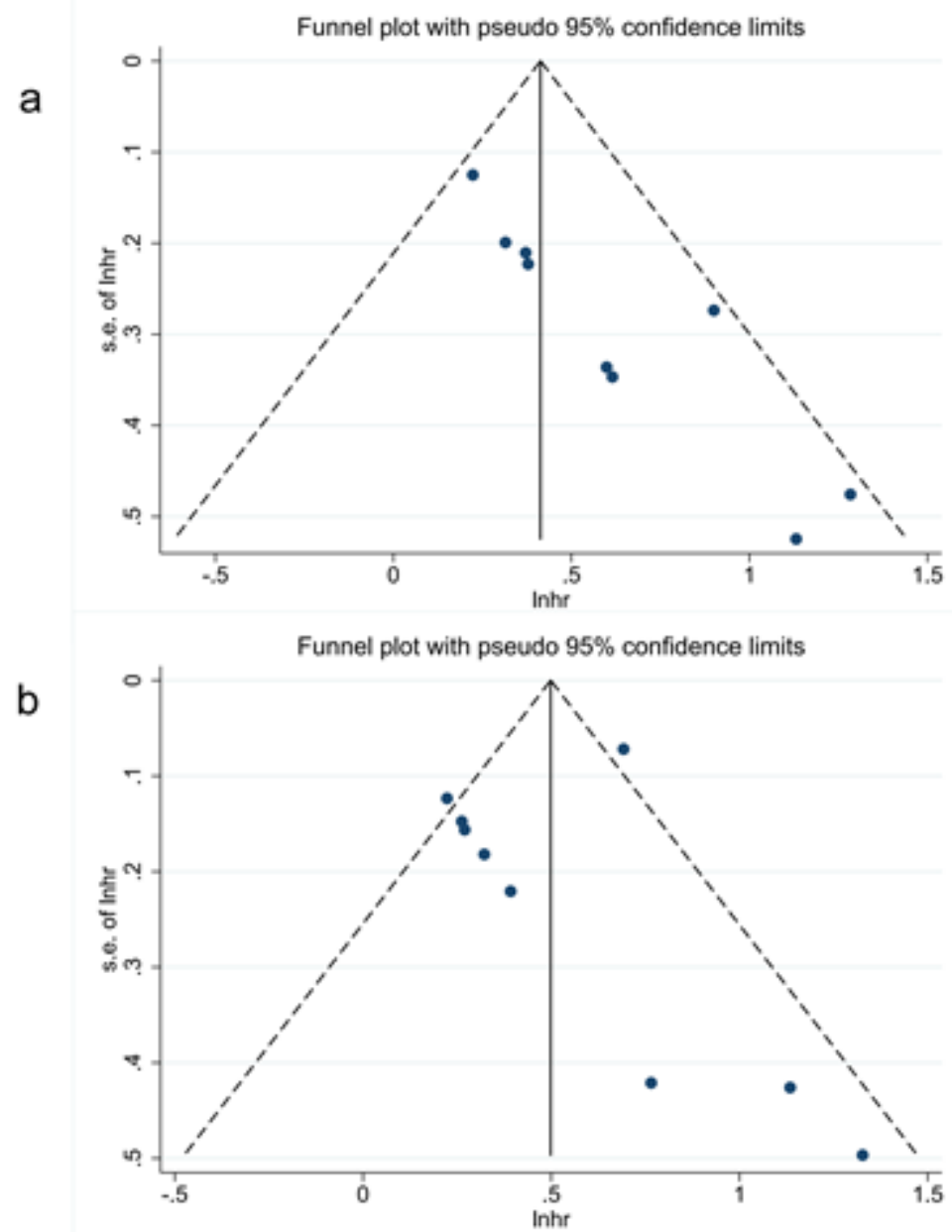

Figure 7

a.Funnel plot of the association between TTS and OS;b.Funnel plot of the association between TTS and RFS/DFS. 
a

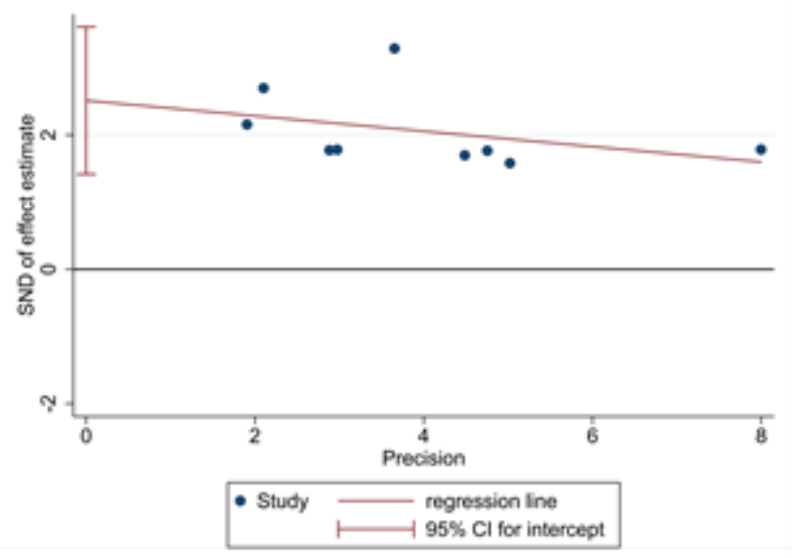

b

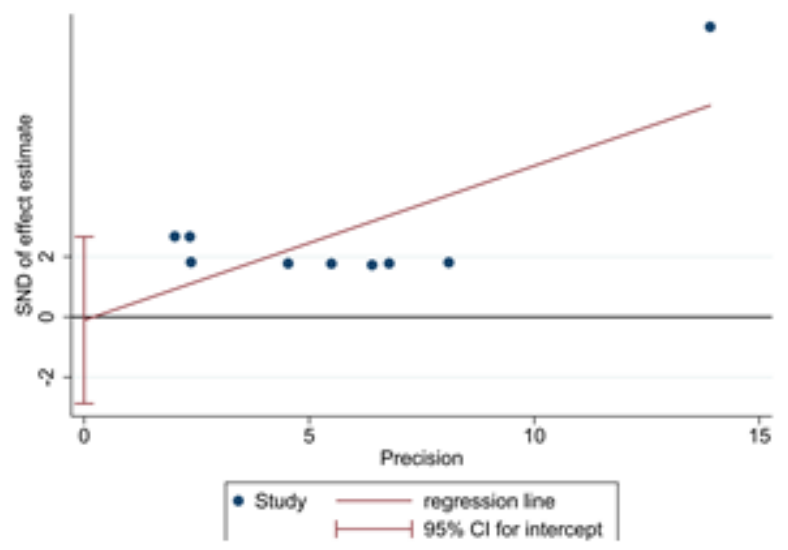

Figure 8

Egger tests for assessment of publication bias (a) for OS and (b) for DFS/RFS.

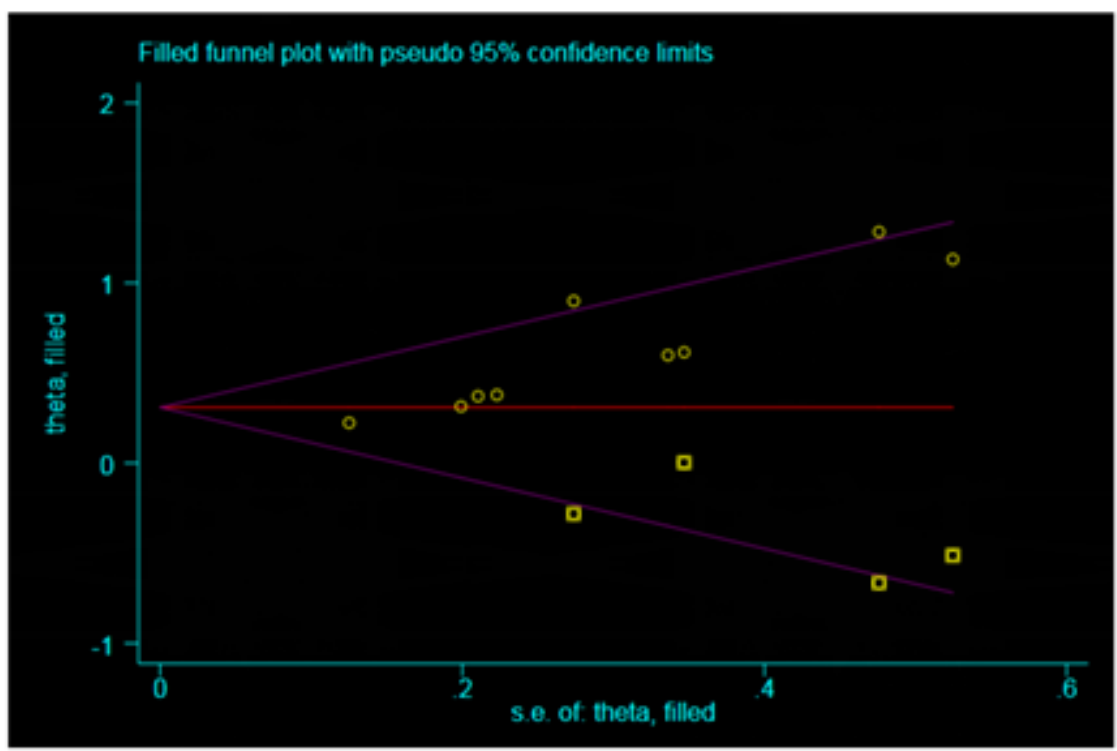

Figure 9 
Publication bias test for the meta-analysis:Trim and fill test for OS,published studies (filled circles) and estimated missing studies (filled diamonds).

\section{Supplementary Files}

This is a list of supplementary files associated with this preprint. Click to download.

- Table1.pdf

- Table2.pdf 